\title{
Effectiveness of Herbicides on Major Aquatic Weed Species of Dalpatsagar Reservoir, Jagdalpur
}

\author{
Subhash Soni*, Prerna Jaiswal and Devid Kumar
}

S. G. College of Agriculture and Research Station, IGKV, Jagdalpur 494005, C.G., India

*Corresponding author

\section{A B S T R A C T}

\section{Keywords}

Effectiveness of herbicide, Chemical herbicide, Phytotoxicity symptoms

\section{Article Info}

\section{Accepted:}

17 January 2021

Available Online:

10 February 2021
Dalpatsagar reservoir is the biggest and oldest water bodies in Jagdalpur city of Chhattisgarh state. The water body was severely infested with different species of aquatic weeds throughout the year since last decade and it modified the structure of habitat and influenced the aquatic ecosystem. The physico-chemical properties of Dalpatsagar reservoir was also changed with season year after year that influenced the water quality and species richness in aquatic ecosystem. In order to reduce the economic, environmental, and social impacts of aquatic weed plants, different management measures was used but most of the cases use of chemical herbicide is necessary to suppress the aggressive nuisance of vegetation. Present study aims to evaluate the effectiveness of Glyphosate, 2,4-D, and Paraquat to control the different species of aquatic macrophytes. An experiment was installed at Dalpatsagar reservoir Jagdalpur, in $31^{\text {st }}$ December 2019 and effectiveness of control was evaluated at $5,10,15,20,25$ and 30 days after spray. The experiment was conducted in Randomized Complete Block Design (RCBD) with three replications comprising seven treatments viz. Glyphosate (41 SL), Paraquat (24 SL) and 2,4-D (amine salt 58\% SL) at 2.00 and 1.00 litre/ha with each herbicides including Absolute control. The herbicides were mixed with sticker (Latron AG-98) and sprayed in three replications by power spray machine (1HP HTP MAK ASPEE) mounted on the boat. At higher dose of Glyhosate @ 2.00 litre/ha had lesser day to yellowing, necrosis and dying than other dose of herbicide treatments, similarly 2,4-D @ 2.00 litre/ha showed the minimum day to appearing phyto-toxicity symptom in all weed species. The reduction of weed density $\left(\right.$ Plant $\left./ \mathrm{m}^{2}\right)$ and weed dry weight $\left(\mathrm{g} / \mathrm{m}^{2}\right)$ at 20 DAS was maximum with Glyphosate @ 2.00 litre/ha followed by 2,4-D @ 2.00 litre/ha. Weed control efficiency was significantly higher in Pistia stratiotes $(81.27 \%)$ and Nelumba nucifera $(81.26 \%)$ with glyphosate@2.00 litre/ha at 5 and 20 DAS. Mean Weed control efficiency was higher (63.80\%) with glyphosate @ 2.00 litre/ha among all weed species followed by 2,4-D @ 2.00 litre/ha. This can be proved that 2,4-D @ 2.00 litre/ha was much effective herbicides to control aquatic weed population than other herbicides followed by 2,4-D @ 2.00 litre/ha. 


\section{Introduction}

Aquatic plant play a key role in any aquatic ecosystem by performing in food chain function as a part of primary producer, protection of habitat and pond ecosystem, removal of organic and toxic compound from wetland and temporal \& spatial changes in diversity of pond ecosystem. So the wetland is a most productive ecosystem on earth, they fulfill the critical regulatory function of hydrological process within the watershed (Hernandez and Mitsch, 2007; Palit and Mukherjee, 2010). However, the anthropogenic activities in aquatic ecosystem increase the nutrient availability which increases the eutrophication and biomass production in pond. Therefore, this condition lead to decrease the species richness, quality standard and reduce the capacity and durability of wetland ecosystem. In order to different aquatic weed management practices the uses of chemical herbicide is an important tools. In most of the cases use of chemical herbicide is necessary to suppress the aggressive nuisance of vegetation. However, the herbicide is applied as a last option in weed suppression system.

In India for controlling various type of submerged and floating weed spices commonly several chemical herbicide like 2,4$\mathrm{D}$, glyphosate, metsulfuron methyl, paraquat etc. are used in a water bodies (Varshney et al., 2008). Different doses of 2,4-D amine salt, 2,4-D Na salt, 2,4-D ester and glyphosate are much effective against floating weed they kill weed within 4 to 11 days (Dutta and Kumar, 2012). Herbicide paraquat, 2,4-D and glyphosate used at the first stage growth of water hyacinth influenced the water quality parameter (Kaur, 2003). Generally water contamination is not occur unless there is considerable run off from the bank of vegetation into the water (Newbold, 1975), and also 2,4-D reduce $50 \%$ or more bottom organisms after one week of application (Walkerm, 1962). Glyphosate herbicide is used to control noxious aquatic weed and algal bloom (Siemering et al., 2008). After the application of glyphosate they reduced 70 to $80 \%$ of weed population within 10 to 20 days (Yirefu and Zekarias, 2009). This herbicide is more effective when applied in aquatic ecosystem then the agriculture or non aquatic use (Duke, 1988). Paraquat chemical herbicide is strongly cationic that persists in water approximately 8 to 11 days (Blackburn and Weldon, 1963). Concentrated form of paraquat is very poisonous so the rate of application for normal use during active growth phase is too low and it killed both type of macrophyte floating and emergent (Newbold, 1975), but the available chemical generally kill all the plant rather than targeted plant (Hershner and Havens, 2008). Based on environment and system application rate varies and herbicidal efficiency dependent on the specific herbicide and their formulation (Masser et al., 2013, Whetstone, 2004).

Thus, the greater knowledge about the chemical control of different aquatic plants can contribute to the decision making on the management of them, which occupy the banks of water bodies, with a high potential of invasion and environmental dynamic change of biotic and abiotic components of aquatic ecosystems. This study aimed to evaluate the effectiveness of the herbicides glyphosate, 2,4-D, and paraquat to control of Eichhornia crassipes, Pistia stratiotes, Ipomoea aquatica, Nelumbo nucifera, Alternanthera philoxeroides, Nymphaea rubra and Cyperus javanicus

\section{Materials and Methods}

Experiment was installed in $31^{\text {st }}$ December 2019 at Dalpatsagar reservoir situated at Jagadalpur city Chhattishgarh. Dalpatsagar reservoir situated in between $19^{\circ} 5^{\prime} 41^{\prime \prime} \mathrm{N}$ and 
$82^{\circ} 0$ ' $43^{\prime \prime}$ E with elevation of $563 \mathrm{~m} \mathrm{MSL}$, the water body was severely infested with many type of aquatic weeds. The water body is located north-west of Jagdalpur city with draining rainwater in the reservoir due to slope towards and the situational dynamics of weeds in the water body discriminates the aquatics throughout year. The experiment consists of three replications with seven treatments that were laid out in Randomized Complete Block Design (RCBD) by piling bamboo poles at measured distance and one bot net was used to confined the aquatic weeds in particular treatments. The treatments comprised of $\mathrm{T}_{1}$ : Glyphosate 41 SL @ 2.0 litre/ha, $\mathrm{T}_{2}$ : Glyphosate 41 SL @ 1.0 litre/ha, T : Paraquat 24 SL @ 2.0 kg/ha, T4: Paraquat 24 SL@ 1.0 $\mathrm{kg} / \mathrm{ha}, \mathrm{T}_{5}: 2$, 4-D (amine salt 58\%) @ 2.0 litre/ha, $\mathrm{T}_{6}:$ 2, 4-D (amine salt 58\%) @ 1.0 litre/ha and $\mathrm{T}_{7}$ : Absolute control (herbicide use restricted). Application of herbicides was done over weed mat dissolving into 500 litre water and added sticker $20 \mathrm{ml}$ (Latron AG98). The chemical spray was done with power spray machine (1HP HTP MAK ASPEE) mounted on the boat. The experimental area was equally divided into 21 plots of $5 \mathrm{~m}^{2}$ by piling bamboo poles at corners of each plot and separated by net. The gap between replication was 3 meter. Herbicide spraying was done on 31 December 2019. After spray of herbicides, weed biomass was loosened and appearance of phyto-toxicity symptoms of herbicides on different weed species was recorded with number of days to yellowing, necrosis and dying of individual weed species. Different weed species response against different herbicide doses with respect to their appearance symptoms of toxicity was considered as yellowing, necrosis and dying. The effectiveness of visual control was performed in RCBD design with one control plot and observation recorded in 5, 10, 15, 20, 25 and 30 days after application. The performance of herbicides was observed visually through the appearance of phyto- toxicity symptoms on the weed plants. Weed density (plants $/ \mathrm{m}^{2}$ ) and Weed dry weight $\left(\mathrm{g} / \mathrm{m}^{2}\right)$ was recorded after oven drying at $60 \pm 50 \mathrm{C}$ for 72 hours and weed control efficiency was calculated with standard method. Data on density and dry weight of weeds were transformed using square root transformation $(\sqrt{x+0.5})$ before statistical analysis as suggested by Panse and Sukhatme (1967). Observed data studies were subjected to statistical analysis as per the guidelines of Gomez and Gomez (1984).

\section{Results and Discussion}

\section{Appearance of phyto-toxicity symptoms by the effect of applied herbicides on weed plants}

\section{Days to yellowing}

Appearance of yellowing on aquatic weeds by the phyto-toxic symptoms of herbicides showed (Table 1) significant difference with respect to herbicides and their dose. The maximum days to yellowing was notice 7 days in Cyperus javanicus with lower dose of Paraquat@1.0 litre/ha, while minimum 5 days took to turn yellowing was noticed in Ipomoea aquatic Alternanthera philoxeroides and Nymphaea rubra when Glyphosate was applied at the rate of 2.00 litre/ha. The Paraquat @ $1.00 \mathrm{~kg} / \mathrm{ha}$ showed significant difference in yellowing at 6 to 7 days while the early symptom of yellowing was observed (5.20 days) in Eichhornia crassipes with Glyphosate @ 2.00 litre/ha. So the application of higher dose of Glyphosate, and 2,4-D are systemic herbicides which lead to little delay yellowing compared to Paraquat, is contact herbicides(Datta 2009).

\section{Days to necrosis}

Appearance of necrosis symptoms in aquatic weed was also observed (Table 2). The 
necrosis symptoms showed the highest 13.33 days under Paraquat @ $1.00 \mathrm{~kg} / \mathrm{ha}$ on Cyperus javanicus and lowest 8.00 days with Ipomoea aquatica under higher dose of Glyphosate @ 2.00 litre/ha. Glyphosate @ 2.00 litre/ha treated plot showed earlier symptoms of necrosis on Eichhornia crassipes, Pistia stratiotes, Ipomoea aquatica, Nelumbo nucifera, Alternanthera philoxeroides, Nymphaea rubra and Cyperus javanicus followed by 2,4-D @ 2.00 litre/ha on same weed species. But in case of Pistia stratiotes, Ipomoea aquatica, Alternanthera philoxeroides, Nymphaea rubra and Cyperus javanicus were at par with higher dose of Paraquat@2.00 kg/ha in showing necrosis symptos, the highest days to develop necrosis was noticed with lower dose of Paraquat applied $1.00 \mathrm{~kg} / \mathrm{ha}$.

\section{Days to dying}

The symptoms of dying after necrosis followed almost similar trend as followed in necrosis of different weed species. There was significant difference in symptoms of dying weed in dalpatsagar reservoir during observation (Table 3). Dying of aquatic weed due to effect of different herbicides was observed and found higher numerical value of days to appearance of dying. The higher days to dying symptoms on weed is noticed when application of Paraquat @ $1.00 \mathrm{~kg} / \mathrm{ha}$ with Cyperus javanicus (22 Days) and lowest value noted with Nymphaea rubra (14.27 days) under Glyphosate 2.00 litre/ha as whole.

Application of Glyphosate @ 2.00 litre/ha was found more effective in showing weeds death with all types of weed species, which was significantly reduce population in the water body and similar effectiveness of developing dead symptoms on weeds was seen with application of 2,4-D @ 2.00 litre/ha considering all weed species under the present study except Cyperus javanicus.

\section{Weed density}

Weed density was significantly influenced by different doses of herbicide application at 20 days after spray (DAS). Aquatic weed population was not significantly influenced by applying herbicides at 5 DAS (Days After Spray) (Table 4). At 5 DAS, Glyphosate @ 2.00 litre/ha had lower density among all weed species and higher density was noticed with 2,4-D@1.00 litre/ha. At 20 DAS (Table 5), herbicide treatment was found significantly difference in density of weed species. The highest weed density was recorded with absolute control (T7) for all weed species and the lowest weed density (plants $/ \mathrm{m}^{2}$ ) of Eichhornia crassipes (2.4), Pistia stratiotes (1.1), Ipomoea aquatic (1.3), Nelumbo nucifera (0.53), Alternanthera philoxeroides (1.53), and Nymphaea rubra (0.86) were recorded with Glyphosate @ 2.00 litre/ha, which was significantly reduced the weed density as compared to other treatments.

\section{Dry weight of weeds}

Weeds Dry weight at 5 DAS (Days After Spray) were no significant difference was observed (Table 6). The dry matter reduction was highest under Glyphosate @ 2.00 litre/ ha in Ipomoea aquatica, while the lowest reduction of dry matter in Eichhornia crassipes (16.45 $\mathrm{g}_{\text {plant }}{ }^{-1}$ ) was recorded with obsolute control (T7). At 20 DAS, weed dry weight was significantly differed with application of herbicide at different dose on different weed species (Table 7). The highest dry weight was observed in absolute control. The highest reduction of dry weight (g) Eichhornia crassipes (4.46g), Pistia stratiotes (1.41g), Ipomoea aquatic (1.63g), Nelumbo nucifera $(1.94 \mathrm{~g})$, Alternanthera philoxeroides (2.13g), and Nymphaea rubra (2.31g) with Glyphosate @ 2.00 litre/ ha followed by 2,4-D @ 2.00 litre/ha. These results are in agreement of those of Chinnusamy et al., (2012). 


\section{Weed control efficiency}

Weed control efficiency was significantly higher in Pistia stratiotes $(81.27 \%)$ and Nelumba nucifera $(81.26 \%)$ with Glyphosate @ 2.00 litre/ha whereas the lowest weed control efficiency was observed (Table 8) in Alternanthera philoxeroides $(3.93 \%)$ under Paraquat at lower dose $(1 \mathrm{~kg} / \mathrm{ha})$ on 20 DAS. At 5 and 20 DAS, Glyphosate @ 2.00 litre/ha gave higher $(63.80 \%)$ weed control efficiency followed by 2,4-D @ 2.00 litre/ha with response to all the weed species. The lowest weed control efficiency was observed with lower dose of Paraquat $(1.00 \mathrm{~kg} / \mathrm{ha})$. The weed control efficiency was increased with Eichhornia crassipes (30.03 and 78.01), Pistia stratioides (65.27 and 81.27) and Ipomoea aquatica (51.21 and $81.26 \%$ ) at 5 and 20 DAS, respectively with Glyphosate @ 2.00 litre/ha (Soni et al., 2020).
Phyto-toxicity symptoms of herbicides viz., days to yellowing, necrosis and dying on different weed species represented the effectiveness of herbicide to control weed plants. At higher dose of Glyhosate @ 2.00 litre/ha had lesser day to yellowing, necrosis and dying than other dose of herbicide treatments, similarly 2,4-D @ 2.00 litre/ha showed the minimum day to appearing phtotoxicity symptom in all weed species. Lower dose of Glyhosate @ 1.00 and Paraquat @ $2.00 \mathrm{~kg} / \mathrm{ha}$ found to be significant variation which took more days to appear toxicity symptoms on weeds. Maximum days to appear phyoto-toxicity symptoms was noticed with lower dose of 2,4-D @ 1.00 litre/ha and Paraquat @ $1.00 \mathrm{~kg} / \mathrm{ha}$ which was more days to complete control of weed as compared to other treatment.

Table.1 Effect of herbicide on major weed species with respect of days to yellowing

\begin{tabular}{|c|c|c|c|c|c|c|c|}
\hline Treatment & \multicolumn{6}{|c|}{ Yellowing of Major Weed flora on Dalpatsagar reservoir (Days) } \\
\cline { 2 - 9 } & $\begin{array}{c}\text { Eichhornia } \\
\text { crassipes }\end{array}$ & $\begin{array}{c}\text { Pistia } \\
\text { stratiotes }\end{array}$ & $\begin{array}{c}\text { Ipomoea } \\
\text { aquatica }\end{array}$ & $\begin{array}{c}\text { Nelumbo } \\
\text { nucifera }\end{array}$ & $\begin{array}{c}\text { Alternanthera } \\
\text { philoxeroides }\end{array}$ & $\begin{array}{c}\text { Nymphaea } \\
\text { rubra }\end{array}$ & $\begin{array}{c}\text { Cyperus } \\
\text { javanicus }\end{array}$ \\
\hline $\mathbf{T}_{\mathbf{1}}$ & 5.20 & 5.27 & 5.00 & 5.40 & 5.00 & 5.00 & 6.07 \\
\hline $\mathbf{T}_{\mathbf{2}}$ & 5.40 & 5.60 & 5.73 & 6.00 & 5.50 & 5.87 & 7.20 \\
\hline $\mathbf{T}_{\mathbf{3}}$ & 5.40 & 5.40 & 5.73 & 5.80 & 5.40 & 5.80 & 6.85 \\
\hline $\mathbf{T}_{\mathbf{4}}$ & 6.00 & 6.57 & 6.33 & 6.60 & 6.73 & 7.20 & 7.27 \\
\hline $\mathbf{T}_{\mathbf{5}}$ & 5.40 & 5.40 & 5.00 & 5.40 & 5.38 & 5.67 & 6.80 \\
\hline $\mathbf{T}_{\mathbf{6}}$ & 5.60 & 6.20 & 6.00 & 6.40 & 5.80 & 6.40 & 7.20 \\
\hline $\mathbf{S E m} \pm$ & $\mathbf{0 . 2 7}$ & $\mathbf{0 . 1 2}$ & $\mathbf{0 . 1 0}$ & $\mathbf{0 . 2 3}$ & $\mathbf{0 . 3 9}$ & $\mathbf{0 . 2 3}$ & $\mathbf{0 . 2 7}$ \\
\hline $\begin{array}{c}\mathbf{C D} \\
(\mathbf{P = 0 . 0 5}\end{array}$ & $\mathbf{0 . 5 9}$ & $\mathbf{0 . 3 7}$ & $\mathbf{0 . 3 2}$ & $\mathbf{0 . 7 3}$ & $\mathbf{N S}$ & $\mathbf{0 . 8 3}$ & $\mathbf{N S}$ \\
\hline
\end{tabular}

T1-Glyphosate @ 2.0 litre/ha, T2- Glyphosate @ 1.0 litre/ha, T3- paraquat @ 2.0 kg/ha, T4- paraquat @ 1.0 kg/ha, T5-2,4-D @ 2 litre/ha, T6-2,4-D @ 1 litre/ha,

* In T7- Absolute control plot herbicide use restricted 
Table.2 Effect of herbicide on major weed species with respect of days to Necrosis

\begin{tabular}{|c|c|c|c|c|c|c|c|}
\hline Treatment & \multicolumn{6}{|c|}{ Necrosis of Major Weed flora on Dalpatsagar reservoir (Days) } \\
\cline { 2 - 9 } & $\begin{array}{c}\text { Eichhornia } \\
\text { crassipes }\end{array}$ & $\begin{array}{c}\text { Pistia } \\
\text { stratiotes }\end{array}$ & $\begin{array}{c}\text { Ipomoea } \\
\text { aquatica }\end{array}$ & $\begin{array}{c}\text { Nelumbo } \\
\text { nucifera }\end{array}$ & $\begin{array}{c}\text { Alternanthera } \\
\text { philoxeroides }\end{array}$ & $\begin{array}{c}\text { Nymphaea } \\
\text { rubra }\end{array}$ & $\begin{array}{c}\text { Cyperus } \\
\text { javanicus }\end{array}$ \\
\hline $\mathbf{T}_{\mathbf{1}}$ & 8.80 & 8.80 & 8.00 & 9.20 & 8.40 & 9.87 & 9.87 \\
\hline $\mathbf{T}_{\mathbf{2}}$ & 9.38 & 10.00 & 9.73 & 11.60 & 9.60 & 10.87 & 12.40 \\
\hline $\mathbf{T}_{\mathbf{3}}$ & 9.20 & 9.67 & 8.67 & 10.40 & 8.80 & 10.13 & 11.60 \\
\hline $\mathbf{T}_{\mathbf{4}}$ & 10.80 & 12.40 & 10.73 & 12.07 & 10.73 & 12.38 & 13.33 \\
\hline $\mathbf{T}_{\mathbf{5}}$ & 8.82 & 9.62 & 8.63 & 9.60 & 8.82 & 10.00 & 10.13 \\
\hline $\mathbf{T}_{\mathbf{6}}$ & 9.67 & 11.60 & 10.00 & 11.67 & 10.00 & 12.38 & 12.40 \\
\hline $\mathbf{S E m} \pm$ & $\mathbf{0 . 1 3}$ & $\mathbf{0 . 2 8}$ & $\mathbf{0 . 2 2}$ & $\mathbf{0 . 8 2}$ & $\mathbf{0 . 6 8}$ & $\mathbf{0 . 0 8}$ & $\mathbf{0 . 3 8}$ \\
\hline $\begin{array}{c}\mathbf{C D} \\
\mathbf{P = 0 . 0 5}\end{array}$ & $\mathbf{0 . 4 3}$ & $\mathbf{0 . 8 2}$ & $\mathbf{0 . 6 8}$ & $\mathbf{0 . 4 8}$ & $\mathbf{0 . 7 5}$ & $\mathbf{0 . 2 8}$ & $\mathbf{1 . 2 0}$ \\
\hline
\end{tabular}

T1- Glyphosate @ 2.0 litre/ha, T2- Glyphosate @ 1.0 litre/ha, T3- paraquat @ 2.0 kg/ha, T4- paraquat @ 1.0 kg/ha,

T5-2,4-D @ 2 litre/ha, T6-2,4-D @ 1 litre/ha,

* In T7-Absolute control plot herbicide use restricted

Table.3 Effect of herbicide on major weed species with respect of days to Dying

\begin{tabular}{|c|c|c|c|c|c|c|c|c|}
\hline \multirow{2}{*}{ Treatment } & \multicolumn{6}{|c|}{ Dying of Major Weed flora on Dalpatsagar reservoir (Days) } \\
\cline { 2 - 9 } & $\begin{array}{c}\text { Eichhornia } \\
\text { crassipes }\end{array}$ & $\begin{array}{c}\text { Pistia } \\
\text { stratiotes }\end{array}$ & $\begin{array}{c}\text { Ipomoea } \\
\text { aquatica }\end{array}$ & $\begin{array}{c}\text { Nelumbo } \\
\text { nucifera }\end{array}$ & $\begin{array}{c}\text { Alternanthera } \\
\text { philoxeroides }\end{array}$ & $\begin{array}{c}\text { Nymphaea } \\
\text { rubra }\end{array}$ & $\begin{array}{c}\text { Cyperus } \\
\text { javanicus }\end{array}$ \\
\hline $\mathbf{T}_{\mathbf{1}}$ & 14.73 & 15.47 & 14.80 & 16.40 & 14.40 & 14.27 & 17.93 \\
\hline $\mathbf{T}_{\mathbf{2}}$ & 18.27 & 16.67 & 16.73 & 19.60 & 17.73 & 19.87 & 20.93 \\
\hline $\mathbf{T}_{\mathbf{3}}$ & 16.67 & 16.53 & 16.67 & 18.53 & 17.27 & 18.13 & 18.47 \\
\hline $\mathbf{T}_{\mathbf{4}}$ & 18.80 & 18.67 & 19.31 & 20.93 & 18.67 & 21.33 & 21.67 \\
\hline $\mathbf{T}_{\mathbf{5}}$ & 16.13 & 15.60 & 16.20 & 17.07 & 16.73 & 17.07 & 19.40 \\
\hline $\mathbf{T}_{\mathbf{6}}$ & 18.77 & 16.93 & 17.53 & 20.53 & 18.07 & 20.67 & 20.93 \\
\hline $\mathbf{S E m} \pm$ & $\mathbf{0 . 5 6}$ & $\mathbf{0 . 0 6}$ & $\mathbf{0 . 5 7}$ & $\mathbf{0 . 2 1}$ & $\mathbf{0 . 8 6}$ & $\mathbf{0 . 8 9}$ & $\mathbf{0 . 6 2}$ \\
\hline $\mathbf{C D}$ & $\mathbf{1 . 1 5}$ & $\mathbf{0 . 1 9}$ & $\mathbf{1 . 4 3}$ & $\mathbf{0 . 6 9}$ & $\mathbf{2 . 6 2}$ & $\mathbf{2 . 6 8}$ & $\mathbf{1 . 9 6}$ \\
\hline $\mathbf{P = 0 . 0 5}$ & & & & & & & \\
\hline
\end{tabular}

T1-Glyphosate @ 2.0 litre/ha, T2- Glyphosate @ 1.0 litre/ha, T3- paraquat @ 2.0 kg/ha, T4- paraquat @ 1.0 kg/ha, T5-2,4-D @ 2 litre/ha, T6-2,4-D @ 1 litre/ha,

* In T7-Absolute control plot herbicide use restricted 
Table.4 Effect of herbicide on weed density (plants $/ \mathrm{m}^{2}$ ) at 5 days after herbicides application

\begin{tabular}{|c|c|c|c|c|c|c|c|}
\hline Treatment & \multicolumn{7}{|c|}{ Major Weed flora on Dalpatsagar reservoir } \\
\cline { 2 - 8 } & $\begin{array}{c}\text { Eichhornia } \\
\text { crassipes }\end{array}$ & $\begin{array}{c}\text { Pistia } \\
\text { stratiotes }\end{array}$ & $\begin{array}{c}\text { Ipomoea } \\
\text { aquatica }\end{array}$ & $\begin{array}{c}\text { Nelumbo } \\
\text { nucifera }\end{array}$ & $\begin{array}{c}\text { Alternanthera } \\
\text { philoxeroides }\end{array}$ & $\begin{array}{c}\text { Nymphaea } \\
\text { rubra }\end{array}$ & $\begin{array}{c}\text { Cyperus } \\
\text { javanicus }\end{array}$ \\
\hline $\mathbf{T}_{\mathbf{1}}$ & 2.60 & 2.38 & 1.96 & 1.45 & 2.12 & 1.33 & 0.71 \\
& $(6.3)$ & $(6.64)$ & $(3.42)$ & $(1.88)$ & $(4.0)$ & $(1.67)$ & $(0.00)$ \\
\hline $\mathbf{T}_{\mathbf{2}}$ & 2.77 & 2.62 & 2.26 & 1.62 & 2.17 & 1.69 & 1.00 \\
& $(7.2)$ & $(4.2)$ & $(4.66)$ & $(2.21)$ & $(4.33)$ & $(2.55)$ & $(0.66)$ \\
\hline $\mathbf{T}_{\mathbf{3}}$ & 2.74 & 2.46 & 2.24 & 1.52 & 2.16 & 1.65 & 0.71 \\
& $(7.06)$ & $(5.65)$ & $(4.53)$ & $(1.88)$ & $(4.22)$ & $(2.33)$ & $(0.00)$ \\
\hline $\mathbf{T}_{\mathbf{4}}$ & 3.21 & 2.73 & 2.42 & 1.69 & 2.36 & 1.77 & 1.22 \\
& $(9.93)$ & $(7.06)$ & $(5.42)$ & $(2.24)$ & $(5.16)$ & $(2.67)$ & $(1.53)$ \\
\hline $\mathbf{T}_{\mathbf{5}}$ & 2.69 & 2.43 & 2.20 & 1.47 & 2.13 & 1.44 & 0.71 \\
& $(6.83)$ & $(5.54)$ & $(4.55)$ & $(1.74)$ & $(4.21)$ & $(1.89)$ & $(0.00)$ \\
\hline $\mathbf{T}_{\mathbf{6}}$ & 2.83 & 2.84 & 2.37 & 1.64 & 2.33 & 1.74 & 1.10 \\
& $(7.63)$ & $(7.63)$ & $(5.21)$ & $(2.33)$ & $(4.99)$ & $(2.55)$ & $(1.00)$ \\
\hline $\mathbf{T}_{7}$ & $3.46 \checkmark$ & $2.85 \checkmark$ & $2.83 \checkmark$ & $1.70 \checkmark$ & $2.39 \checkmark$ & $2.05 \checkmark$ & $1.44 \checkmark$ \\
& $(11.53)$ & $(7.63)$ & $(7.55)$ & $(2.44)$ & $(5.33)$ & $(3.78)$ & $(2.66)$ \\
\hline $\mathbf{S E m} \pm$ & $\mathbf{0 . 1 8}$ & $\mathbf{0 . 3 6}$ & $\mathbf{0 . 1 8}$ & $\mathbf{0 . 2 3}$ & $\mathbf{0 . 2 2}$ & $\mathbf{0 . 1 8}$ & $\mathbf{0 . 3 9}$ \\
\hline $\mathbf{C D}$ & $\mathbf{0 . 5 5}$ & $\mathbf{N S}$ & $\mathbf{N S}$ & $\mathbf{N S}$ & $\mathbf{N S}$ & $\mathbf{N S}$ & NS \\
\hline$(\mathbf{P}=\mathbf{0 . 0 5})$ & & & & & & & NS \\
\hline
\end{tabular}

T1-Glyphosate @ 2.0 litre/ha, T2- Glyphosate @ 1.0 litre/ha, T3- paraquat @ 2.0 kg/ha, T4- paraquat @ 1.0 kg/ha, T5-2,4-D @ 2 litre/ha, T6- 2,4-D @ 1 litre/ha,T7- Absolute control (herbicide use restricted)

Table.5 Effect of herbicide on weed density (plants $/ \mathrm{m}^{2}$ ) at 20 days after herbicides application

\begin{tabular}{|c|c|c|c|c|c|c|c|}
\hline Treatment & \multicolumn{7}{|c|}{ Major weed flora on Dalpatsagar reservoir } \\
\cline { 2 - 9 } & $\begin{array}{c}\text { Eichhornia } \\
\text { crassipes }\end{array}$ & $\begin{array}{c}\text { Pistia } \\
\text { stratiotes }\end{array}$ & $\begin{array}{c}\text { Ipomoea } \\
\text { aquatica }\end{array}$ & $\begin{array}{c}\text { Nelumbo } \\
\text { nucifera }\end{array}$ & $\begin{array}{c}\text { Alternanthera } \\
\text { philoxeroides }\end{array}$ & $\begin{array}{c}\text { Nymphaea } \\
\text { rubra }\end{array}$ & $\begin{array}{c}\text { Cyperus } \\
\text { javanicus }\end{array}$ \\
\hline $\mathbf{T}_{\mathbf{1}}$ & 1.68 & 1.20 & 1.27 & 0.95 & 1.27 & 1.13 & 0.71 \\
& $(2.4)$ & $(1.1)$ & $(1.3)$ & $(0.53)$ & $(1.53)$ & $(0.86)$ & $(0.00)$ \\
\hline $\mathbf{T}_{\mathbf{2}}$ & 1.75 & 1.26 & 1.41 & 1.27 & 1.46 & 1.27 & 0.77 \\
& $(2.63)$ & $(1.1)$ & $(1.53)$ & $(1.3)$ & $(1.93)$ & $(1.3)$ & $(0.1)$ \\
\hline $\mathbf{T}_{\mathbf{3}}$ & 1.71 & 1.26 & 1.30 & 1.24 & 1.39 & 1.27 & 0.77 \\
& $(2.5)$ & $(1.26)$ & $(1.4)$ & $(1.2)$ & $(1.73)$ & $(1.3)$ & $(0.1)$ \\
\hline $\mathbf{T}_{\mathbf{4}}$ & 1.88 & 1.73 & 1.62 & 1.40 & 1.62 & 1.38 & 0.88 \\
& $(3.06)$ & $(2.63)$ & $(2.2)$ & $(1.53)$ & $(2.2)$ & $(1.43)$ & $(0.33)$ \\
\hline $\mathbf{T}_{\mathbf{5}}$ & 1.69 & 1.24 & 1.27 & 1.12 & 1.35 & 1.21 & 0.71 \\
& $(2.53)$ & $(1.2)$ & $(1.3)$ & $(0.86)$ & $(1.53)$ & $(1.1)$ & $(0.00)$ \\
\hline $\mathbf{T}_{\mathbf{6}}$ & 1.76 & 1.27 & 1.54 & 1.29 & 1.55 & 1.35 & 0.71 \\
& $(2.73)$ & $(1.3)$ & $(2.26)$ & $(1.33)$ & $(1.86)$ & $(1.53)$ & $(0.00)$ \\
\hline $\mathbf{T}_{\mathbf{7}}$ & $3.37 \checkmark$ & $2.58 \checkmark$ & $2.70 \checkmark$ & $1.86 \checkmark$ & $1.86 \checkmark$ & $1.64 \checkmark$ & $1.03 \checkmark$ \\
& $(10.93)$ & $(6.2)$ & $(6.83)$ & $(2.96)$ & $(2.96)$ & $(2.2)$ & $(0.76)$ \\
\hline $\mathbf{S E m} \pm$ & $\mathbf{0 . 0 2}$ & $\mathbf{0 . 0 0 1}$ & $\mathbf{0 . 0 1}$ & $\mathbf{0 . 0 6}$ & $\mathbf{0 . 0 3}$ & $\mathbf{0 . 0 2}$ & $\mathbf{0 . 0 3}$ \\
\hline $\mathbf{C D}(\mathbf{P}=\mathbf{0 . 0 5})$ & $\mathbf{0 . 0 6}$ & $\mathbf{0 . 0 3}$ & $\mathbf{0 . 0 2}$ & $\mathbf{0 . 1 8}$ & $\mathbf{0 . 0 9}$ & $\mathbf{0 . 0 8}$ & $\mathbf{0 . 1 1}$ \\
\hline
\end{tabular}

T1-Glyphosate @ 2.0 litre/ha, T2- Glyphosate @ 1.0 litre/ha, T3- paraquat @ 2.0 kg/ha, T4- paraquat @ 1.0 kg/ha, T5-2,4-D @ 2 litre/ha, T6- 2,4-D @ 1 litre/ha,T7- Absolute control (herbicide use restricted) 
Table.6 Effect of herbicide on weed dry weight (g.) at 5 days after herbicides application

\begin{tabular}{|c|c|c|c|c|c|c|c|}
\hline \multirow{2}{*}{ Treatment } & \multicolumn{7}{|c|}{ Major Weed flora on Dalpatsagar reservoir } \\
\cline { 2 - 8 } & $\begin{array}{c}\text { Eichhornia } \\
\text { crassipes }\end{array}$ & $\begin{array}{c}\text { Pistia } \\
\text { stratiotes }\end{array}$ & $\begin{array}{c}\text { Ipomoea } \\
\text { aquatica }\end{array}$ & $\begin{array}{c}\text { Nelumbo } \\
\text { nucifera }\end{array}$ & $\begin{array}{c}\text { Alternanthera } \\
\text { philoxeroides }\end{array}$ & $\begin{array}{c}\text { Nymphaea } \\
\text { rubra }\end{array}$ & $\begin{array}{c}\text { Cyperus } \\
\text { javanicus }\end{array}$ \\
\hline $\mathbf{T}_{\mathbf{1}}$ & 3.45 & 1.72 & 2.00 & 2.65 & 2.32 & 2.36 & 0.71 \\
& $(11.51)$ & $(2.99)$ & $(3.52)$ & $(8.43)$ & $(4.95)$ & $(6.47)$ & $(0.00)$ \\
\hline $\mathbf{T}_{\mathbf{2}}$ & 3.74 & 2.12 & 2.24 & 3.12 & 2.56 & 3.15 & 1.31 \\
& $(13.62)$ & $(4.12)$ & $(4.51)$ & $(9.39)$ & $(6.1)$ & $(9.84)$ & $(1.94)$ \\
\hline $\mathbf{T}_{\mathbf{3}}$ & 3.49 & 2.10 & 2.16 & 3.08 & 2.47 & 3.12 & 0.71 \\
& $(12.00)$ & $(4.20)$ & $(4.28)$ & $(9.12)$ & $(5.81)$ & $(9.41)$ & $(0.00)$ \\
\hline $\mathbf{T}_{\mathbf{4}}$ & 3.99 & 2.59 & 2.60 & 3.56 & 2.65 & 3.59 & 1.63 \\
& $(15.50)$ & $(6.28)$ & $(6.32)$ & $(12.28)$ & $(6.60)$ & $(12.56) 2$ & $(3.84)$ \\
\hline $\mathbf{T}_{\mathbf{5}}$ & 3.47 & 1.98 & 2.13 & 2.96 & 2.42 & 2.40 & 0.71 \\
& $(11.73)$ & $(4.26)$ & $(4.21)$ & $(8.31)$ & $(5.37)$ & $(6.73)$ & $(0.00)$ \\
\hline $\mathbf{T}_{\mathbf{6}}$ & 3.84 & 2.27 & 2.24 & 3.22 & 2.57 & 3.33 & 1.50 \\
& $(14.43)$ & $(4.86)$ & $(4.62)$ & $(9.94)$ & $(6.23)$ & $(10.86)$ & $(2.98)$ \\
\hline $\mathbf{T}_{\mathbf{7}}$ & $4.10 \checkmark$ & $3.02 \checkmark$ & $2.76 \checkmark$ & $3.61 \checkmark$ & $2.75 \checkmark$ & $3.77 \checkmark$ & $1.78 \checkmark$ \\
& $(16.45)$ & $(8.61)$ & $(7.22)$ & $(12.69)$ & $(7.06)$ & $(13.71)$ & $(4.91)$ \\
\hline $\mathbf{S E m} \pm$ & $\mathbf{0 . 2 2}$ & $\mathbf{0 . 3 6}$ & $\mathbf{0 . 2 0}$ & $\mathbf{0 . 4 1}$ & $\mathbf{0 . 1 6}$ & $\mathbf{0 . 3 7}$ & $\mathbf{0 . 6 8}$ \\
\hline $\mathbf{C D}$ & $\mathbf{N S}$ & $\mathbf{N S}$ & $\mathbf{N S}$ & $\mathbf{N S}$ & $\mathbf{N S}$ & $\mathbf{N S}$ & $\mathbf{N S}$ \\
$(\mathbf{P = 0 . 0 5 )}$ & & & & & & & NS \\
\hline
\end{tabular}

T1- Glyphosate @ 2.0 litre/ha, T2- Glyphosate @ 1.0 litre/ha, T3- paraquat @ 2.0 kg/ha, T4- paraquat @ 1.0 kg/ha,

T5-2,4-D @ 2 litre/ha, T6-2,4-D @ 1 litre/ha,T7- Absolute control (herbicide use restricted)

Table.7 Effect of herbicide on weed dry weight (g.) at 20 days after herbicides application

\begin{tabular}{|c|c|c|c|c|c|c|c|}
\hline \multirow{2}{*}{ Treatment } & \multicolumn{9}{|c|}{ Major Weed flora on Dalpatsagar reservoir } \\
\cline { 2 - 8 } & $\begin{array}{c}\text { Eichhornia } \\
\text { crassipes }\end{array}$ & $\begin{array}{c}\text { Pistia } \\
\text { stratiotes }\end{array}$ & $\begin{array}{c}\text { Ipomoea } \\
\text { aquatica }\end{array}$ & $\begin{array}{c}\text { Nelumbo } \\
\text { nucifera }\end{array}$ & $\begin{array}{c}\text { Alternanthera } \\
\text { philoxeroides }\end{array}$ & $\begin{array}{c}\text { Nymphaea } \\
\text { rubra }\end{array}$ & $\begin{array}{c}\text { Cyperus } \\
\text { javanicus }\end{array}$ \\
\hline $\mathbf{T}_{\mathbf{1}}$ & 2.18 & 1.30 & 1.36 & 1.31 & 1.50 & 1.55 & 0.71 \\
& $(4.46)$ & $(1.41)$ & $(1.63)$ & $(1.94)$ & $(2.13)$ & $(2.31)$ & $(0.00)$ \\
\hline $\mathbf{T}_{\mathbf{2}}$ & 2.31 & 1.55 & 1.55 & 1.84 & 1.67 & 1.97 & 0.71 \\
& $(4.86)$ & $(2.33)$ & $(2.36)$ & $(3.67)$ & $(2.78)$ & $(4.29)$ & $(0.00)$ \\
\hline $\mathbf{T}_{\mathbf{3}}$ & 2.25 & 1.45 & 1.51 & 1.84 & 1.62 & 1.93 & 0.71 \\
& $(4.67)$ & $(1.91)$ & $(2.11)$ & $(3.58)$ & $(2.65)$ & $(4.35)$ & $(0.00)$ \\
\hline $\mathbf{T}_{\mathbf{4}}$ & 2.99 & 1.77 & 2.00 & 2.21 & 2.30 & 2.62 & 1.23 \\
& $(8.52)$ & $(2.67)$ & $(3.55)$ & $(5.63)$ & $(4.89)$ & $(6.49)$ & $(1.56)$ \\
\hline $\mathbf{T}_{\mathbf{5}}$ & 2.25 & 1.31 & 1.49 & 1.76 & 1.60 & 1.76 & 0.71 \\
& $(4.91)$ & $(1.41)$ & $(2.06)$ & $(3.13)$ & $(2.46)$ & $(3.18)$ & $(0.00)$ \\
\hline $\mathbf{T}_{\mathbf{6}}$ & 2.36 & 1.66 & 1.78 & 2.07 & 1.95 & 2.00 & 1.14 \\
& $(5.31)$ & $(2.35)$ & $(2.74)$ & $(3.82)$ & $(3.39)$ & $(4.43)$ & $(1.17)$ \\
\hline $\mathbf{T}_{\mathbf{7}}$ & $3.79 \checkmark$ & $2.83 \checkmark$ & $2.44 \checkmark$ & $3.28 \checkmark$ & $2.34 \checkmark$ & $3.13 \checkmark$ & $1.35 \checkmark$ \\
& $(13.94)$ & $(7.53)$ & $(5.49)$ & $(10.36)$ & $(5.09)$ & $(9.44)$ & $(2.16)$ \\
\hline $\mathbf{S E m} \pm$ & $\mathbf{0 . 2 4}$ & $\mathbf{0 . 3 2}$ & $\mathbf{0 . 3 4}$ & $\mathbf{0 . 5 5}$ & $\mathbf{0 . 3 8}$ & $\mathbf{0 . 5 4}$ & $\mathbf{0 . 0 2}$ \\
\hline $\mathbf{C D}$ & $\mathbf{0 . 7 4}$ & $\mathbf{N S}$ & $\mathbf{N S}$ & $\mathbf{N S}$ & $\mathbf{N S}$ & $\mathbf{N S}$ & $\mathbf{0 . 0 8}$ \\
\hline
\end{tabular}

T1-Glyphosate @ 2.0 litre/ha, T2- Glyphosate @ 1.0 litre/ha, T3- paraquat @ 2.0 kg/ha, T4- paraquat @ 1.0 kg/ha, T5-2,4-D @ 2 litre/ha, T6- 2,4-D @ 1 litre/ha,T7- Absolute control (herbicide use restricted) 
Table.8 Weed control efficiency of different treatments

\begin{tabular}{|c|c|c|c|c|c|c|c|c|c|c|c|c|c|c|c|}
\hline \multirow[t]{3}{*}{ Treatment } & \multicolumn{15}{|c|}{ Weed control efficiency (\%) } \\
\hline & \multicolumn{2}{|c|}{$\begin{array}{c}\text { Eichhornia } \\
\text { crassipes }\end{array}$} & \multicolumn{2}{|c|}{$\begin{array}{c}\text { Pistia } \\
\text { stratiotes }\end{array}$} & \multicolumn{2}{|c|}{$\begin{array}{l}\text { Ipomoea } \\
\text { aquatica }\end{array}$} & \multicolumn{2}{|c|}{$\begin{array}{l}\text { Nelumbo } \\
\text { nucifera }\end{array}$} & \multicolumn{2}{|c|}{$\begin{array}{l}\text { Alternanthera } \\
\text { philoxeroides }\end{array}$} & \multicolumn{2}{|c|}{$\begin{array}{c}\text { Nymphaea } \\
\text { rubra }\end{array}$} & \multicolumn{2}{|c|}{$\begin{array}{l}\text { Cyperus } \\
\text { javanicus }\end{array}$} & \multirow[t]{2}{*}{ Mean } \\
\hline & 5 DAS & $\begin{array}{c}20 \\
\text { DAS }\end{array}$ & $\begin{array}{c}5 \\
\text { DAS }\end{array}$ & $\begin{array}{c}20 \\
\text { DAS }\end{array}$ & $\begin{array}{c}5 \\
\text { DAS }\end{array}$ & $\begin{array}{c}20 \\
\text { DAS }\end{array}$ & $\begin{array}{c}5 \\
\text { DAS }\end{array}$ & $\begin{array}{c}20 \\
\text { DAS }\end{array}$ & $\begin{array}{c}5 \\
\text { DAS }\end{array}$ & $\begin{array}{c}20 \\
\text { DAS }\end{array}$ & $\begin{array}{c}5 \\
\text { DAS }\end{array}$ & $\begin{array}{c}20 \\
\text { DAS }\end{array}$ & 5 DAS & $\begin{array}{c}20 \\
\text { DAS }\end{array}$ & \\
\hline T1 & 30.03 & 78.01 & 65.27 & 81.27 & 51.25 & 70.31 & 33.57 & 81.26 & 29.89 & 58.15 & 52.81 & 75.53 & 76.59 & 76.30 & 63.80 \\
\hline $\mathbf{T} 2$ & 17.20 & 55.14 & 52.15 & 69.06 & 37.53 & 57.01 & 26.00 & 64.58 & 13.60 & 45.38 & 28.23 & 54.56 & 50.49 & 45.37 & 45.45 \\
\hline T3 & 27.05 & 65.50 & 51.22 & 74.63 & 40.72 & 61.57 & 28.13 & 65.44 & 17.71 & 47.94 & 31.36 & 53.92 & 56.19 & 52.78 & 50.51 \\
\hline T4 & 5.78 & 38.88 & 27.06 & 64.54 & 12.47 & 35.34 & 3.23 & 45.66 & 6.52 & 3.93 & 8.39 & 31.25 & 21.79 & 29.63 & 23.89 \\
\hline T5 & 28.69 & 76.78 & 52.52 & 80.27 & 41.69 & 62.48 & 34.52 & 69.79 & 23.94 & 51.67 & 50.91 & 66.31 & 72.89 & 74.35 & 51.70 \\
\hline T6 & 12.28 & 61.91 & 43.55 & 68.79 & 36.01 & 50.09 & 21.67 & 63.13 & 11.76 & 33.40 & 20.79 & 53.07 & 39.31 & 13.43 & 37.80 \\
\hline T7 & 0.00 & 0.00 & 0.00 & 0.00 & 0.00 & 0.00 & 0.00 & 0.00 & 0.00 & 0.00 & 0.00 & 0.00 & 0.00 & 0.00 & 0.00 \\
\hline SEm \pm & 0.75 & 0.38 & 3.54 & 1.67 & 2.65 & 2.20 & 0.37 & 3.98 & 3.06 & 2.57 & 0.61 & 5.44 & 2.87 & 10.95 & \\
\hline $\begin{array}{c}C D \\
(P=0.05)\end{array}$ & 3.01 & 2.51 & 14.05 & 6.64 & 10.53 & 7.84 & 2.44 & 13.83 & 7.18 & 8.22 & 1.93 & 9.32 & 6.81 & 43.52 & \\
\hline
\end{tabular}

$\mathrm{T}_{1}$ : Glyphosate @ 2.0 litre/ha, $\mathrm{T}_{2}$ : Glyphosate @ 1.0 litre/ha, $\mathrm{T}_{3}$ : Paraquat @ 2.0 kg/ha, $\mathrm{T}_{4}$ : Paraquat @ 1.0 kg/ha, T5:2,4-D @ 2 litre/ha, $\mathrm{T}_{6}: 2,4-\mathrm{D} @ 1$ litre/ha, $\mathrm{T}_{7}$ : Absolute control 
Highest weed density was observed with Absolute control at both 5 and 20 DAS (Days After Spray), the reduction of weed density was lowest due to herbicide use restricted on weed population. The maximum density of weed (Plants $\left./ \mathrm{m}^{2}\right)$ was reduced with Glyphosate@ 2.00 litre/ha on Eichhornia crassipes (2.4), Pistia stratiotes (1.1), Ipomoea aquatic (1.3), Nelumbo nucifera (0.53), Alternanthera philoxeroides (1.53), and Nymphaea rubra (0.86). Similarly, 2,4-D @ 2.00 litre/ha reduced weed density significantly. Weed dry weight was also recorded highest at 5 and 20 DAS (Days After Spray) with Absolute control treatment. The reduction of dry weight was also found same trend as weed density, reduction of dry weight (g) was highest with Eichhornia crassipes (4.46g), Pistia stratiotes (1.41g), Ipomoea aquatic (1.63g), Nelumbo nucifera (1.94g), Alternanthera philoxeroides (2.13g), and Nymphaea rubra (2.31g) with Glyphosate @ 2.00 litre/ ha followed by 2,4-D @ 2.00 litre/ha.Weed control efficiency was significantly higher in Pistia stratiotes (81.27\%) and Nelumba nucifera (81.26\%) with Glyphosate @ 2.00 litre/ha at 5 and 20 DAS. Mean Weed control efficiency was higher (63.80\%) with Glyphosate @ 2.00 litre/ha among all weed species followed by 2,4-D@2.00 litre/ha. This can be proved that Glyphosate @ 2.00 litre/ha was much effective herbicides to control aquatic weed population than other herbicides followed by 2,4-D@2.00 litre/ha.

\section{References}

Blackburn, R. O. and Weldon, L. W. 1963. Results of three years' testing diquat as an aquatic herbicide in Florida. Proc. sth. Weed Control Conf., 16th, 365.

Chinnusamy, C., Janaki, P., Murali Arthanari, P. and Muthukrishnan, P. 2012. Effect of Post-emergence Herbicides on Water hyacinth (Eichhornia crassipes)- Tank Culture Experiments Pak. J. Weed Sci.
Res., 18: 105-111

Datta.S. 2009. Aquatic Weeds and Their Management for Fisheries https://www.researchgate.net/publication /258931408 p22.

Duke, S. O. 1988. Glyphosate. In: Herbicides, Chemistry, degradation and mode of action, Kearney, P.C. \& Kaufman, D.D. (Ed.), 1-70.

Dutta, S. and Kumar, A. 2012. Effect of Some Herbicide In Controlling The Floating Aquatic Weed, Diversification of Aquaculture P 301-313.

Gomez, K.A. and Gomez, A.A. 1984. Statistical procedures for Agricultural Research. A Willey.

Hernandez, M.E. and Mitsch, W.J. 2007. Denitrifcation in created riverine wetlands: influence of hydrology and season. Ecol Eng 30:78-88.

Hershner, C., and K.J. Havens. 2008. Managing invasive aquatic plants in a changing system: Strategic consideration of ecosystem services. Conservation Biol. 22: 544-550.

Kaur, S. 2003. Impact of herbicides on water quality and fish mortality in relation to water hyacinth control. M.Sc. thesis, NRC Weed Science, Jabalpur, India.

Masser, M.P., Murphy, T.R. and Shelton, J.L. 2013. Aquatic weed management: herbicides. Southern regional aquaculture center.(http://srac.tamu.edu/index.cfm/e vent/getFactSheet/whichfactsheet/66/.ht $\mathrm{ml}$ )

Newbold, C., 1975. Herbicide In Aquatic System, Biol. Consev 27 Applied Science Publisher Ltd.- England.

Palit, D., and Mukherjee, A. 2010. Characterization of Physico-chemical properties of water and soil in Lalb and hafresh water wetland in Birbhum District West Bengal. Ecology Environment and Conservation 15(4) 239-245.

Panse, V.G. and Sukhatme, P.V. 1967. Statistical methods of agricultural workers. Indian Council of Agricultural 
Research, New Delhi

Siemering, G., Hayworth, J. and Greenfield, B. 2008. Assessment of potential herbicide impacts to California aquatic ecosystems. Arch. Environ. Contam. Toxicol., 55, 415-431.

Soni., Subhash., Pradhan, A., Thakur, A.K., Chandrakar, T. and Singh, D.P. 2020. Effect of physico-chemical properties and weed management practices on weed dynamics of Dalpatsagar reservoir, Jagdalpur. Int.J.Curr. Microbiol.App.Sci (2020) 9(10): 747-755

Varshney Jay G, Sushilkumar and Mishra J.S. 2008. Current status of aquatic weeds and their management in India. Proceedings th of Tal: (Eds. Sengupta
M. and Dalwani R.) The 12 World Lake Conference, Jiapur: 1039- 1045.

Walker, C. R. 1962. Toxicological effects of several herbicides to bottom dwelling fish food organisms in Missouri ponds. Res. Rep. N. cent. Weed Control Conf., 18th, 104-5.

Whetstone, J. M. 2004. Aquatic weed control overview. Clemson Cooperative Extension. (http://www.clemson.edu/ extension/hgic/plants/pdf/hgic1714.pdf)

Yirefu, F. and Zekarias, Y. 2009. Evaluation of Some Herbicide against Water Hyacinth (Eichhornia crassipes Mart.[solm.]) at Wonji- Shoa. Proc. Ethiop. Sugar. Ind. Bienn. Conf., 1: 61-68.

\section{How to cite this article:}

Subhash Soni, Prerna Jaiswal and Devid Kumar. 2021. Effectiveness of Herbicides on Major Aquatic Weed Species of Dalpatsagar Reservoir, Jagdalpur. Int.J.Curr.Microbiol.App.Sci. 10(02): 1966-1976. doi: https://doi.org/10.20546/ijcmas.2021.1002.236 